\section{Long-horned Beetles (Coleoptera: Cerambycidae) and Tortoise Beetles (Chrysomelidae: Cassidinae) of Tripura, northeastern India with some new additions}

\section{B.K. Agarwala ${ }^{1} \&$ Partha Pratim Bhattacharjee ${ }^{2}$}

1,2 Ecology and Biodiversity Laboratories, Department of Zoology, Tripura University, Suryamaninagar, Tripura 799022, India Email: ${ }^{1}$ bagarwala00@gmail.com (corresponding author), ${ }^{2}$ bhattacharjee.pp1977@gmail.com

Members of the family Cerambycidae are commonly known as Longicorn or Long-horned Beetles. This family includes a vast assemblage of phytophagous and xylophagous insects. This is one of the largest families of Coleoptera and contains more than 35,000 species under 4,000 genera in 11 subfamilies (Lawrence 1982). The family, though predominant in tropics, is distributed throughout the world. The number of cerambycid species recorded from India is about 1500 (Beeson 1941; Breuning 1960-62, 1963a, 1963b, 1964, 1965, 1966) including 13 species reported from Tripura (Mukhopadhyay \&

Date of publication (online): 26 October 2012

Date of publication (print): 26 October 2012

ISSN 0974-7907 (online) | 0974-7893 (print)

Editor: Anonymity requested

Manuscript details:

Ms \# 02951

Received 19 September 2011

Final received 18 April 2012

Finally accepted 25 August 2012

Citation: Agarwala, B.K. \& P.P. Bhattacharjee (2012). Long-horned Beetles (Coleoptera: Cerambycidae) and Tortoise Beetles (Chrysomelidae: Cassidinae) of Tripura, northeastern India with some new additions. Journal of Threatened Taxa 4(13): 3223-3227.

Copyright: @ B.K. Agarwala \& Partha Pratim Bhattacharjee 2012. Creative Commons Attribution 3.0 Unported License. JoTT allows unrestricted use of this article in any medium for non-profit purposes, reproduction and distribution by providing adequate credit to the authors and the source of publication.

Acknowledgements: Authors are thankful to ICAR, New Delhi for financia support through National Project on Insect Biosystematics and to Dr. V.V. Ramamurthy, Indian Agriculture Research Institute, New Delhi for encouragements. We also wish to express our gratitude to Dr. H.V. Ghate, Department of Zoology, Modern College of Arts, Science and Commerce, Pune for identifying / confirming some of the species reported here.

ZooBank urn:Isid:zoobank.org:pub:5CBB5733-6BAE-4064-B44546A9DFBFB7B7

\section{OPEN ACCESS | FREE DOWNLOAD (C) (i) ৫}

Biswas 2002). Several new species have been described since the above studies, and many are being documented in Indian territory for the first time (e.g. Ghate et al. 2006, 2011) and so the number of cerambycid species found in India may have changed.

Members of the subfamily Cassidinae (Chrysomelidae) are popularly known as Tortoise Beetles. There are 2,760 species of tortoise beetles known in the world so far (Borowiec 1999) including 450 species recorded from India and four species from Tripura (Basu 2002). Jacoby (1908), Maulik (1919, 1926), Scherer (1969), Takizawa (1980), Borowiec \& Takizawa (1991) and Borowiec (1999) have produced monographic works on Indian Chrysomelidae, including tortoise beetles. In this communication, 11 species of Cerambycidae and seven species of Cassidinae beetles are reported as new records from the state of Tripura in India.

\section{Study site}

Tripura, one of the border states of northeastern India, lies between $22^{0} 55^{\prime}-24^{0} 32^{\prime} \mathrm{N} \& 91^{0} 21^{\prime}-92^{0} 16^{\prime} \mathrm{E}$. The state has an area of $10,492 \mathrm{~km}^{2}$ with $53.62 \%$ of area under forest cover. Landscape of the state comprise of low hills covered with moist deciduous forests dominated by Shorea and Tectona trees with thick understorey, undulating hillocks covered with secondary forests dominated by Dipterocarpus trees and bushes, and agricultural plains dominated by paddy crop with rain-fed rivers and patches of plantation crops (jute, tea, rubber) and fruit trees (pineapples, mango, cashew nuts and jackfruits) (Chakraborty 1989, 2003). Thus, the landscape of Tripura is very heterogeneous which provides edge effects and diverse habitat types for flora and fauna.

\section{Methods}

Collections were made during January 2007-December 2010 by frequent visits to forested and cultivated habitats in different parts of the state. Collected specimens were identified to species level following key characters provided by Gahan (1906), Maulik (1919, 1926), Cherepanov (1990), Mukhopadhyay \& Biswas (2000), Basu (2002) and Mukhopadhyay \& Halder (2004) and also by 
comparison with the identified materials available in the ecology and biodiversity laboratories, Department of Zoology, Tripura University where voucher specimens of species reported here are kept.

\section{Discussion}

In the present study, 19 species of Cerambycidae belonging to three subfamilies were recorded. The subfamily Lamiinae is found to be dominant with 11 species, followed by Cerambycinae with seven species, and one species belonged to the subfamily Prioninae. Eleven of these species are reported here for the first time from Tripura (Table 1, Images 1-12). In case of tortoise beetles, eight species were recorded, of which seven species are reported as new records from Tripura (Table 2, Images 13-20). Considering the lack of studies on the Cerambycidae and Cassidinae insect biodiversity in this region, the findings are very significant for the understanding of insect biodiversity in Tripura State and providing baseline data.

\section{REFERENCES}

Basu, C.R. (2002). Insecta: Coleoptera: Chrysomelidae. Fauna of Tripura, State Fauna Series, Zoological Survey of India 7: 143-164.

Beeson, C.F.C. (1941). The Ecology and Control of Forest Insects of India and the Adjoining Countries. Government of India, 767pp.

Borowiec, L. (1999). A World Catalogue of the Cassidinae (Coleoptera: Chrysomelidae). Biologia Silesiae, Wroclaw, Poland, 476pp.

Borowiec, L. \& H. Takizawa (1991). Notes on Chrysomelid beetles (Coleoptera) of India and its neighbouring areas. Japanese Journal of Entomology 59: 637-654.

Breuning, S. (1960-62). Revision systematique Des especes du genre Oberea Mulsant du globe. Frustula Entomologica (Pt. 1, 2, 3): 232pp.

Breuning, S. (1963a). Bestimmungstabella der Lamiiden Triben nebst Revision der Pteropliini der asiatischen Region (Col. Ceramb.) 111 Teil. Entomologischen Arbeiten ausdem Museum G. Frey 14: 168-251.

Breuning, S. (1963b). Bestimmungstabelle der Lamiiden Triben nebst Revision der Pteropliini der asiatischen Region (Col. Ceramb.) 111 Teil. Entomologischen Arbeiten ausdem Museum G. Frey 14: 466-537.

Breuning, S. (1964). Revision der Apomecynini der asiatischaustralischen Region. Entomologische Abhandlungen Museum fur Tierkunde in Dresden 30: 528pp.
Breuning, S. (1965). Revision der 35 Gattung der Pteropliini der asiatischen Region (Col. Cerambycidae). Entomologische Arbeiten aus dem Museum G Frey 16: 161-472.

Breuning, S. (1966). Revision der Agapanthini der eurasiatisch australischen Region (Coleoptera: Cerambycidae). Entomologischen Abhandlungen Museum fur Tierkunde in Dresden 34 (1): 144pp.

Chakraborty, N.K. (1989). Useful plants of Tripura jute fields. Journal of Economic and Taxonomic Botany 13: 357-366.

Chakraborty, N.K. (2003). Tripurar Upakari Agacha. Jnan Bichitra Prakashani, Agartala, 160pp.

Cherepanov, A.I. (1990). Cerambycidae of Northern Asia: Lamiinae - Vol. 3, Part 2. Amerind Publishing Co. Pvt. Ltd., New Delhi, 324pp.

Gahan, C.J. (1906). The Fauna of British India including Ceylon and Burma. Coleoptera: Cerambycidae. Taylor and Francis, London, 329pp.

Ghate, H.V., M.H. Kichloo \& M. Arif (2006). First record of a cerambycid beetle Purpuricenus kabakovi Miroshnikov \& Lobanov from Kashmir, northern India. Zoos' Prints Journal 21(11): 2473-2474.

Ghate, H.V., C.A. Viraktamath \& R. Sundararaj (2011). First report of a Cerambycid beetle (Capnolymma cingalensis) from India. Taprobanica 3(2): 104-106.

Jacoby, M. (1908). The Fauna of British India including Ceylon and Burma. Coleoptera: Chrysomelidae. Taylor and Francis, London, 1: xx+554pp.

Lawrence, J.F. (1982). Coleoptera, pp. 482-553. In: Parker, S. (ed.). Synopsis and Classification of Living Organisms. McGraw Hill, New York.

Maulik, S. (1919). The Fauna of British India Including Ceylon and Burma. Coleoptera: Chrysomelidae (Cassidinae and Hispinae). Taylor and Francis, London, xii+440pp.

Maulik, S. (1926). The Fauna of British India, including Ceylon and Burma. Coleoptera: Chrysomelidae (Chrysomelinae and Halticinae). Taylor and Francis, London, xiii+442pp.

Mukhopadhyay, P. \& S. Biswas (2000). Coleoptera: Cerambycidae, pp. 41-67. In: Director (ed.). Fauna of Meghalaya, State Fauna Series 4 (Part 5). Zoological Survey of India Publication.

Mukhopadhyay, P. \& S. Biswas (2002). Coleoptera: Cerambycidae, pp. 139-142. In: Director (ed.). Fauna of Tripura, State Fauna Series 7 (Part 3). Zoological Survey of India Publication.

Mukhopadhyay, P. \& S.K. Halder (2004). Insecta: Coleoptera: Cerambycidae, pp. 421-431. In: Director (ed.). Fauna of Manipur, State Fauna Series 10 (Part 2). Zoological Survey of India Publication.

Scherer, G. (1969). Die Alticinae des indischen Subkotinentes (Coleoptera: Chrysomelidae). Pacific Insects Monographs 22: $1-251$.

Takizawa, H.(1980). Immature stages of some Indian Cassidinae (Coleoptera: Chrysomelidae). Insecta Matsumurana (New Series) 21: 19-48. 

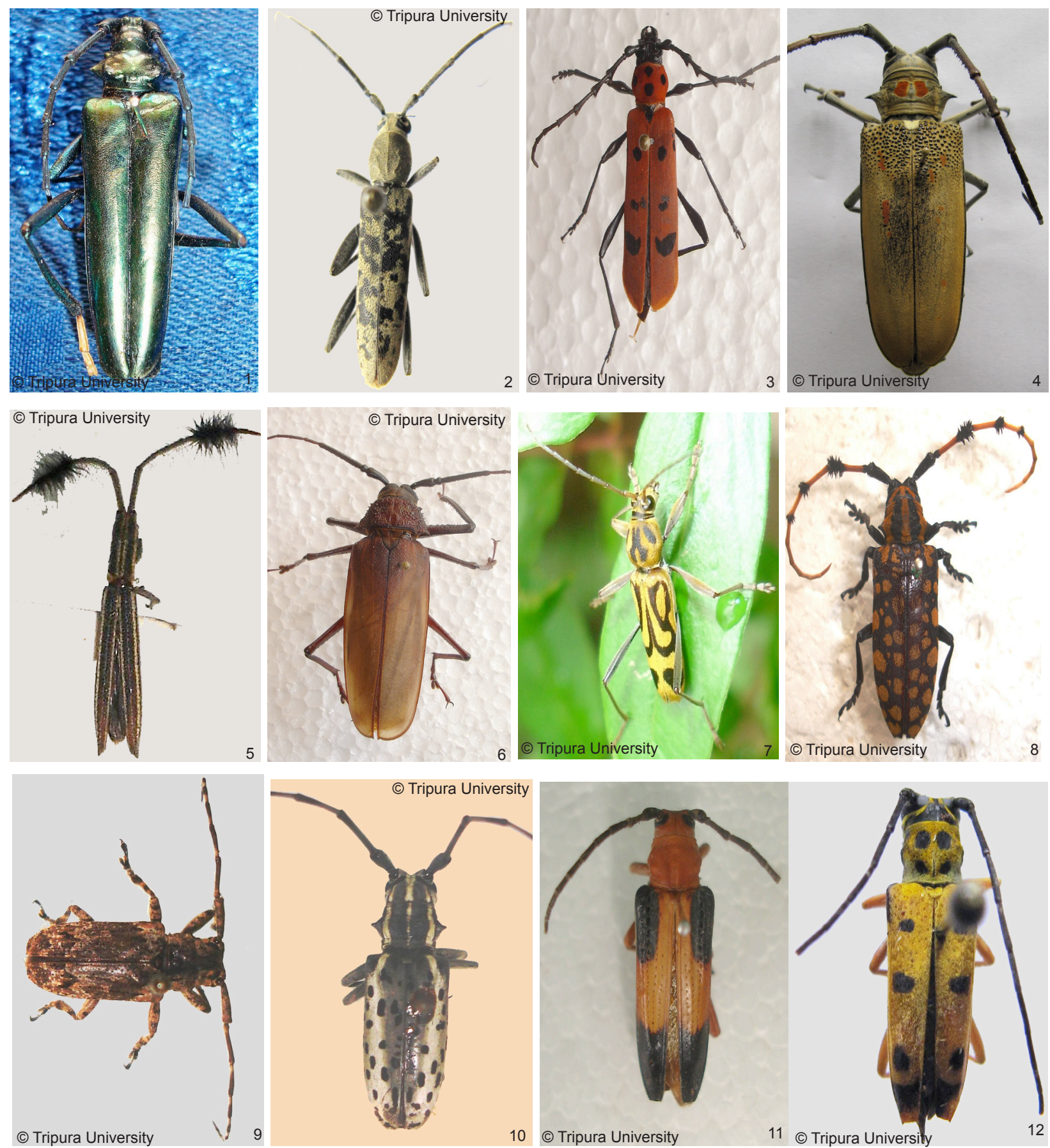

Images 1-12. Cerambycidae beetles.

1 - Aphrodisium cantori (Hope), 2 - Oemospila maculipennis Gahan, 3 - Rosalia decempunctata (Westwood), 4- Batocera numitor Newman, 5 - Eucomatocera vittata White, 6 - Bander pascoei Lansberge, 7 - Chlorophorus annularis F., 8 - Aristobia testudo Voet, 9 - Coptops aedificator F., 10 - Epepeotes uncinatus Gahan, 11 - Stibara tetraspilota Hope, 12 - Glenea flava Jordon. 
Table 1. Records of Cerambycidae beetles of Tripura State

\begin{tabular}{|c|c|c|c|c|}
\hline \multirow[t]{2}{*}{ Species } & \multicolumn{2}{|c|}{ Distribution in Tripura } & \multirow[t]{2}{*}{ Reference No.* } & \multirow[t]{2}{*}{ Remark } \\
\hline & $\begin{array}{l}\text { Earlier recorded by } \\
\text { Mukhopadhyay \& Biswas } \\
(2002)\end{array}$ & $\begin{array}{l}\text { Records of new } \\
\text { distribution }\end{array}$ & & \\
\hline \multicolumn{5}{|l|}{ Superfamily: Cerambycoidea } \\
\hline \multicolumn{5}{|l|}{ Family: Cerambycidae } \\
\hline \multicolumn{5}{|l|}{ Subfamily: Cerambycinae } \\
\hline 1. Anubis inermis (White) & Abhya, s. Tripura & - & ZSI collection $^{+}$ & - \\
\hline 2. Aphrodisium cantori (Hope) & - & Trishna WS, s. Tripura & 2673 & New Record \\
\hline 3. Chelidonium gibbicole (White) & Santibazar, s. Tripura & Trishna WS, s. Tripura & 2674 & - \\
\hline $\begin{array}{l}\text { 4. Chlorophorus annularis F. } \\
\text { (=Caloclytus annularis F.) }\end{array}$ & - & Jampui, n. Tripura & 2675 & New Record \\
\hline 5. Noserius sp. & Sikaribari, n. Tripura & - & ZSI collection $^{+}$ & - \\
\hline 6. Oemospila maculipennis Gahan & - & Jampui, n. Tripura & 3125 & New Record \\
\hline 7. Rosalia decempunctata (Westwood) & - & Trishna WS, s. Tripura & 2668 & New Record \\
\hline 8. Stromatium barbatum $\mathrm{F}$. & Ambasa, d. Tripura & $\begin{array}{l}\text { Bhubanban, w. Tripura } \\
\text { Trishna WS, s. Tripura }\end{array}$ & 2672 & - \\
\hline 9. Xystrocera globosa Olivier & $\begin{array}{l}\text { Belonia, s. Tripura, Belonia, } \\
\text { s. Tripura }\end{array}$ & Trishna WS, s. Tripura & 2679 & - \\
\hline \multicolumn{5}{|l|}{ Subfamily: Lamiinae } \\
\hline 10. Aristobia testudo Voet & - & Suryamaninagar, w. Tripura & 207 & New Record \\
\hline 11. Batocera numitor Newman & - & Jampui, n. Tripura & 3126 & New Record \\
\hline 12. Centrura sp. & Chimbagan, n. Tripura & - & ZSI collection ${ }^{+}$ & - \\
\hline 13. Coptops aedificator F. & - & Trishna WS, s. Tripura & 3127 & New Record \\
\hline 14. Epepeotes uncinatus Gahan & - & Maharani, s. Tripura & 3130 & New Record \\
\hline 15. Eucomatocera vittata White & - & Subalsing, w. Tripura & 2681 & New Record \\
\hline 16. Glenea indiana Thomson & $\begin{array}{l}\text { Baramura, w. Tripura, } \\
\text { Abhya, s. Tripura }\end{array}$ & Mirza, s. Tripura & 178 & - \\
\hline 17. Glenea assimilis Gahan & $\begin{array}{l}\text { Thakachera, Ameupur, s. } \\
\text { Tripura }\end{array}$ & - & $\mathrm{ZSI}_{\text {collection }}{ }^{+}$ & - \\
\hline 18. Glenea flava Jordan & - & Jampui, n. Tripura & 3128 & - \\
\hline 19. Macrochenus guerini White & Kungmura, s. Tripura & Maharani; s. Tripura & 3129 & - \\
\hline 20. Olenecamptus bilobus F. & Agartala, w. Tripura & Trishna WS, s. Tripura & 2666 & - \\
\hline 21. Olenecamptus sp. & Sonamura, s. Tripura & Trishna WS, s. Tripura & 2667 & - \\
\hline 22. Oberea posticata Boheman & Abhya, s. Tripura & - & ZSI collection ${ }^{+}$ & - \\
\hline 23. Oberea sp. & Baramura, w. Tripura & - & ZSI collection $^{+}$ & - \\
\hline 24. Stibara tetraspilota Hope & - & Trishna WS, s. Tripura & 2680 & New Record \\
\hline \multicolumn{5}{|l|}{ Subfamily: Prioninae } \\
\hline 25. Bander pascoei Lansberge & - & Trishna WS, s. Tripura & 2665 & New Record \\
\hline
\end{tabular}

w. Tripura = west Tripura District; s. Tripura = south Tripura District; $n$. Tripura = north Tripura District; d. Tripura = Dhalai Tripura District; Trishna WS = Trishna Wildlife Sanctuary; * Reference collection number (Coleoptera / Cerambycidae- \#) of Insect Biosystematics Centre, Department of Zoology, Tripura University; ${ }^{+}$As per the information documented in Mukhopadhyay \& Biswas (2002). 

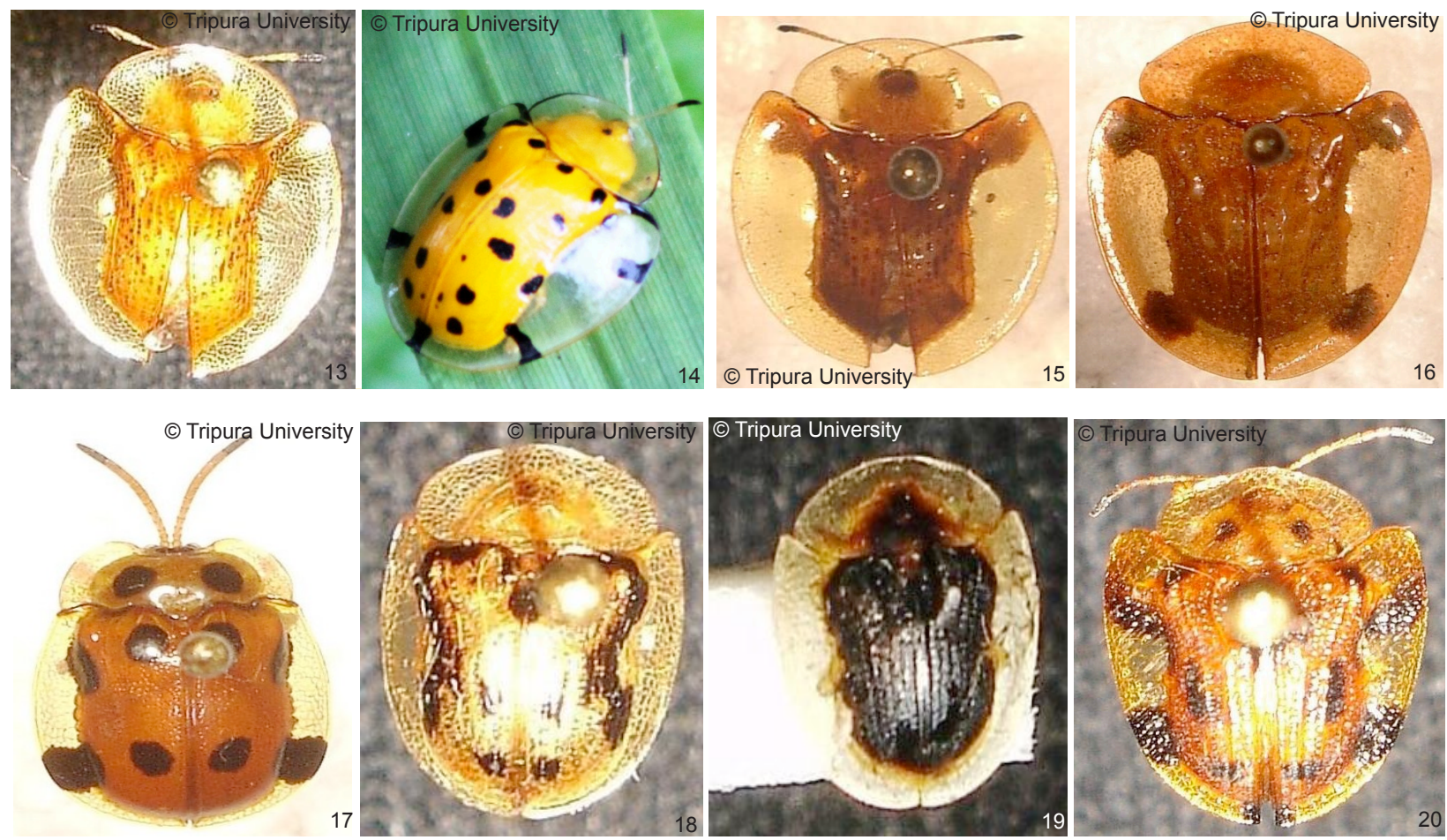

Images 13-20. Cassidinae beetles.

13 - Aspidimorpha dorsata (F.), 14 - Aspidimorpha miliaris (F.), 15 - Aspidimorpha furcata (Thunberg), 16 - Aspidimorpha sanctaecrucis (F.), 17 - Basiprionota decemmaculata (Boheman), 18 - Cassida sp., 19 - Cassida flavoscutata Spaeth, 20 - Laccoptera nepalensis Boheman

Table 2. Records of Cassidinae beetles of Tripura State

\begin{tabular}{|c|c|c|c|c|}
\hline \multirow[t]{2}{*}{ Species } & \multicolumn{2}{|c|}{ Distribution in Tripura } & \multirow[t]{2}{*}{ Reference No.* } & \multirow[t]{2}{*}{ Remark } \\
\hline & $\begin{array}{l}\text { Earlier recorded by } \\
\text { Basu (2002) }\end{array}$ & $\begin{array}{l}\text { Record of new } \\
\text { distribution }\end{array}$ & & \\
\hline \multicolumn{5}{|l|}{ Superfamily: Chrysomeloidea } \\
\hline \multicolumn{5}{|l|}{ Family: Chrysomelidae } \\
\hline \multicolumn{5}{|l|}{ Subfamily: Cassidinae } \\
\hline 1. Aspidimorpha dorsata (F.) & - & Jampui, n. Tripura & 3231 & New Record \\
\hline 2. Aspidimorpha furcata (Thunberg) & - & Salema, d. Tripura & 76 & New Record \\
\hline 3. Aspidimorpha miliaris (F.) & - & Jampui, n. Tripura & 3232 & New Record \\
\hline 4. Aspidimorpha sanctaecrucis (F.) & - & Pandabpur, w. Tripura & 79 & New Record \\
\hline 5. Basiprionota decemmaculata (Boheman) & - & Jampui, n. Tripura & 3233 & New Record \\
\hline 6. Cassida catenata Boheman & $\begin{array}{l}\text { Bagma, Shalgara, } \\
\text { s. Tripura }\end{array}$ & - & ZSI collection $^{+}$ & - \\
\hline 7. Cassida circumdata Herbst & Barpathari, s. Tripura & - & ZSI collection $^{+}$ & - \\
\hline 8. Cassida flavoscutata Spaeth & - & Bagabasa, s. Tripura & 77 & New Record \\
\hline 9. Cassida sp. & - & Jampui, n. Tripura & 3234 & - \\
\hline 10. Chiridopsis scalaris (Weber) & Belonia, s. Tripura & - & ZSI collection $^{+}$ & - \\
\hline 11. Laccoptera nepalensis Boheman & - & Jampui, n. Tripura & 3235 & New Record \\
\hline 12. Laccoptera quadrimaculata (Thunberg) & $\begin{array}{l}\text { Ambassa, Dulubari, } \\
\text { d. Tripura }\end{array}$ & - & ZSI collection $^{+}$ & - \\
\hline
\end{tabular}

w. Tripura = west Tripura; s. Tripura = south Tripura; $n$. Tripura = north Tripura; d. Tripura = dhalai Tripura; * Reference collection number (Coleoptera $/$ Chrysomelidae- \#) of Insect Biosystematics Centre, Department of Zoology, Tripura University; ${ }^{+}$As per the information documented in Basu (2002) 